\title{
Experimental resin-modified calcium-silicate cement containing N-(2- hydroxyethyl) acrylamide monomer for pulp tissue engineering
}

\author{
MARIANO S. PEDANO ${ }^{1 *}$, KUMIKO YOSHIHARA $^{2,3}{ }^{*}, \mathrm{XIN} \mathrm{LI}^{1}$, BERNARDO CAMARGO $^{1}$, \\ KIRSTEN VAN LANDUYT ${ }^{1}$, BART VAN MEERBEEK ${ }^{1}$
}

${ }^{1} \mathrm{KU}$ Leuven (University of Leuven), Department of Oral Health Sciences, BIOMAT - Biomaterials Research group \& UZ Leuven (University Hospitals Leuven), Dentistry, Leuven, Belgium.

${ }^{2}$ National Institute of Advanced Industrial Science and Technology (AIST), Health Research Institute, 2217-14 Hayashi-Cho, Takamaysu, Kagawa, 761-0395, Japan.

${ }^{3}$ Okayama University, Graduate School of Medicine, Dentistry and Pharmaceutical Sciences, Department of Pathology \& Experimental Medicine, 2-5-1 Shikata-cho, Kita-ku, Okayama, 700-8558, Japan.

* Equal first-author contribution.

Running title: HEAA acrylamide-based pulp-capping agent.

\section{Keywords:}

Bioceramics; Vital pulp therapy; Human vital tooth-culture model; Acrylamide monomer, Micro-CT

Corresponding author: Prof. Dr. Bart Van Meerbeek, KU Leuven (University of Leuven), Department of Oral Health Sciences, BIOMAT, Campus St.-Raphaël, Kapucijnenvoer 7 block a box 7001, BE-3000 Leuven, Belgium; +32-16-337587; bart.vanmeerbeek@kuleuven.be 


\section{ABSTRACT}

Aim. Our study aimed to measure (1) the flexural strength, (2) shear bond strength to dentin, (3) pH, and (4) calcium ( $\mathrm{Ca}$ ) release of a series of innovative resin-modified calcium-silicate pulp-capping cements (Rm-CSCs). Using an ex-vivo human vital tooth-culture model, we additionally assessed (5) their pulp-healing initiation when brought in direct contact with human dental pulp tissue.

Methodology. Three experimental Rm-CSCs, being referred to 'Exp_HEAA', 'Exp_GDM' and 'Exp_HEAA/GDM', contained either 20wt\% N-(2-hydroxyethyl) acrylamide (HEAA), 20wt\% glycerol dimethacrylate (GDM) or 10wt\% HEAA plus 10wt\% GDM, added to a common base composition consisting of $25 \mathrm{wt} \%$ urethane dimethacrylate (UDMA), 10wt\% 4-methacryloxyethyl trimellitate anhydride (4-MET), and 5wt\% N,N'-\{[(2-acrylamido-2-[(3-acrylamidopropoxy)methyl] propane-1,3diyl)bis(oxy)]bis-(propane-1,3-diyl)\}diacrylamide (FAM-401). As Ca source and radiopacifier, 37wt\% tricalcium silicate powder (TCS) and 3wt\% zirconium oxide $\left(\mathrm{ZrO}_{2}\right)$ were respectively added.

Results. All three experimental Rm-CSCs revealed a significantly higher flexural strength and shear bond strength to dentin $(p<0.05)$ than the commercial reference Rm-CSC TheraCal LC (Bisco). Exp_HEAA presented with a significantly higher Ca release and pH at 24 hrs compared with the other Rm-CSCs $(p<0.05)$. At 1 week, the Ca release and pH of Exp_HEAA and Exp_HEAA/GDM was significantly higher than those of Exp_GDM and TheraCal LC $(p<0.05)$. Using the ex-vivo human vital tooth-culture model, Exp_HEAA revealed pulp-healing initiation capacity as documented by nestin and collagen-I expression.

Conclusions. Depending on the formulation, the innovative Rm-CSCs performed favorably for primary properties of relevance regarding pulp capping, this more specifically in terms of flexural strength, bond strength to dentin, as well as alkaline $\mathrm{pH}$ and Ca release. However, only Exp_HEAA revealed pulphealing initiation in direct contact with human dental pulp tissue in the ex-vivo human vital toothculture model. This promising outcome for Exp_HEAA should be attributed to the combined use of (1) a novel hydrophilic acrylamide monomer, enabling sufficient polymerization while maintaining adequate hydrophilicity, with (2) the functional monomer 4-MET, possessing chemical bonding potential to dentin, and (3) tricalcium silicate powder to achieve an alkaline $\mathrm{pH}$ and to release $\mathrm{Ca}$ in a sufficient and controlled way. 


\section{Introduction}

Resin-free hydraulic calcium-silicate cements (hCSCs) join calcium-hydroxide $(\mathrm{CH})$ as gold-standard materials for vital pulp therapy $[1,2]$. However, the poor physical properties of $\mathrm{CH}$, as well as the long setting time and difficult handling properties of hCSCs remain major shortcomings $[3,4]$.

To solve these issues of classic pulp-capping agents, resin-modified calcium-silicate cements (RmCSCS), like TheraCal LC (Bisco, Schaumburg, IL, USA) or Biocal Cap (Harvard, Hoppegarten, Germany), have recently been marketed $[5,6]$. A methacrylate resin-based composition renders better physicomechanical properties, while an added photo-initiation system enables setting-on-command, providing a more user-friendly application [5]. A resin basis furthermore offers the possibility to add functional monomers with dentin-bonding potential. Nevertheless, currently available Rm-CSCs do still not possess the optimum material characteristics pulp-capping agents should meet. As primary property, pulp-capping agents should be bioactive towards human dental pulp cells (HDPCs) by releasing a relatively high amount of calcium (Ca) to the adjacent pulp tissue $[7,8]$. Moreover, as these materials are usually placed in an inflamed environment surrounded by bacteria, a high $\mathrm{pH}$ with alkalinizing and disinfecting properties is highly desirable [9-11]. Ideally, pulp-capping agents should effectively bond to the dentinal tissue around the pulp exposure, this to adequately seal the pulpal wound. In light of these primary property requirements, the commercial Rm-CSC TheraCal LC (Bisco) falls somewhat short for weak physico-mechanical properties and relatively low bonding potential to dentin $[12,13]$. Furthermore, its $\mathrm{pH}$ and $\mathrm{Ca}$ release are still rather low, as compared with those obtained with resin-free hCSCs [14]. Besides, TheraCal LC (Bisco) has been shown to be cytotoxic towards HDPCs. This may be the reason why its capacity to form a reparative dentin bridge upon capping is rather limited as compared to other resin-free pulp-capping agents [15-17].

Recently, our group introduced two Rm-CSCs containing bisphenol A-glycidyl methacrylate (BisGMA), glycerol dimethacrylate (GDM), N-(2-hydroxyethyl) acrylamide (HEAA), the functional monomer 4-methacryloxyethyl trimellitate anhydride (4-MET) and the novel acrylamide monomer $N, N^{\prime}-\{[(2-$ acrylamido-2-[(3-acrylamidopropoxy)methyl]propane-1,3-diyl)bis(oxy)]bis-(propane-1,3-diyl)\} diacrylamide (FAM-401) [18]. These experimental Rm-CSC formulations revealed improved cytocompatibility, high polymerization efficiency, good flexural strength and adequate bond strength to dentin [18], when compared to TheraCal LC (Bisco). However, due to alleged bisphenol A (BPA) concerns associated with Bis-GMA-containing materials, three other innovative Rm-CSC formulations on basis of urethane dimethacrylate (UDMA) were designed, synthesized and mutually tested on their pulp-capping potential in this research work. By combining a strong di-methacrylate crosslinking monomer, like UDMA, with a functional co-monomer, like 4-MET, better physico-mechanical properties and bond strength to dentin are targeted [19]. Specific acrylamide monomers were added 
to ensure adequate hydrophilicity, while also facilitating the bioactive potential. By adding pure tricalcium-silicate powder (TCS), a high pH and substantial Ca release were aimed [20, 21].

Therefore, the objectives of this study were (1) to measure the flexural strength, (2) shear bond strength to dentin, (3) pH, and (4) Ca release of a series of three innovative Rm-CSCs. Using an ex-vivo human vital tooth-culture model, we additionally assessed (5) their pulp-healing initiation when brought in direct contact with human dental pulp tissue. The null-hypotheses tested were that the new experimentally developed pulp-capping agents do not have improved flexural strength (1), increased shear bond strength to dentin (2), higher $\mathrm{pH}$ (3), nor higher Ca release (4), as compared to the commercial Rm-CSC TheraCal LC (Bisco) that served as reference. Finally, no pulp-healing initiation (5) was expected upon pulp capping with the Rm-CSCs using the ex-vivo human vital tooth-culture model.

\section{Material and methods}

\section{Preparation of experimental materials}

The composition of the experimental and commercial Rm-CSCs is detailed in Table 1. To prepare three experimental Rm-CSC formulations, two different hydrophilic monomers were selected: (1) N-(2hydroxyethyl) acrylamide (HEAA) and (2) glycerol dimethacrylate (GDM) (Fig. 1). The three Rm-CSCs, being referred to as Exp_HEAA, Exp_GDM and Exp_HEAA/GDM, possessed a similar base composition, which consisted in order of decreasing concentration of 25wt\% UDMA, 10wt\% 4-MET, 5wt\% FAM-401, to which either 20wt\% HEAA, $20 w t \%$ GDM or a combination of $10 w t \%$ HEAA and $10 w t \%$ GDM was added, respectively (Table 1). The Ca source selected was pure TCS powder (37wt\%) (Mineral Research Processing, Meyzieu, France), while $3 \mathrm{wt} \% \mathrm{ZrO}_{2}$ (Schott, Mainz, Germany) was added as radiopacifier. The commercial Rm-CSC TheraCal (Bisco) served as test reference.

\section{Three-point bending flexural strength}

For each cement, standardized cement bars ( $n=10$ per Rm-CSC/condition), measuring $2 \times 2 \times 25 \mathrm{~mm}$, were prepared using Teflon molds. The Rm-CSC bar was light cured for $60 \mathrm{~s}$ in total using a calibrated LED light-curing unit (G-Light Prima II Plus, GC, Tokyo, Japan) with the light guide moved across three adjacent overlapping spots, which received $20 \mathrm{~s}$ light exposure each, so to ensure adequate polymerization of the whole bar. Each specimen was stored at $37^{\circ} \mathrm{C}$ in $100 \%$ humidity for $24 \mathrm{~h}$. Next, the cement bars were either tested or kept for three additional weeks in distilled water at $37^{\circ} \mathrm{C}$ prior to being subjected to a three-point bending test with a $20-\mathrm{mm}$ span and load speed of $0.5 \mathrm{~mm} / \mathrm{min}$ (Model 5565, Instron, Canton, MA, USA), as outlined in the ISO 9917-2 (1996) standard. The flexural strength was determined from the highest stress measured at fracture. 


\section{Shear bond strength to dentin}

Recently extracted human third molars ( $n=10 / R m-C S C$ ) [approved by the institute's Committee for Medical Ethics (file number: 1606-020)] were embedded in epoxy resin (Epofix, Struers, Ballerup, Denmark), upon which the dentin surface was exposed/ground using 600-grit SiC-paper (WTCC-S, Nikken, Fukuyama, Japan) to produce a standard smear layer. Teflon tubes with a 3.6-mm diameter and 2-mm thickness were used to confine the dentin surface area the Rm-CSCs were exposed to. Following fixation of the Teflon mold to dentin using a mounting jig, the experimental and reference Rm-CSCs were applied, covered with a plastic mylar sheet and light cured for $40 \mathrm{~s}$ (G-Light Prima II Plus, $\mathrm{GC}$ ). The specimens were next stored at $37^{\circ} \mathrm{C}$ in $100 \%$ humidity for $24 \mathrm{~h}$ prior to being subjected to a shear bond-strength test. The specimens were mounted into the testing machine (Autograph DCS2000, Shimadzu, Kyoto, Japan) and subjected to shear stress at a crosshead speed of $0.5 \mathrm{~mm} / \mathrm{min}$. The force was applied via a flat 1-mm-thick shearing blade in a direction parallel to the $\mathrm{Rm}$-CSC/dentin interface. Stress at failure was calculated and recorded as the shear bond strength. All fractured specimens were analyzed utilizing a light microscope (SMZ-10, Nikon, Tokyo, Japan) at 4x magnification to determine the mode of fracture.

\section{$\mathrm{pH}$ and Ca release}

Test cylinders (8-mm diameter, 2-mm height) were prepared cured and stored at $37^{\circ} \mathrm{C}$ in $100 \%$ humidity for $24 \mathrm{~h}$. Each Rm-CSC cylinder was next immersed in $5 \mathrm{ml}$ distilled water, which was collected and refreshed after 1 day, 4 and 7 days. The collected solution's $\mathrm{pH}$ was measured using a pH meter (Laqua, Horiba, Kyoto, Japan), while Ca release was analyzed using Inductively Coupled Plasma Atomic Emission Spectrometry (ICP-AES; VISTA Pro, SII, Chiba, Japan). Prior to analysis, the solution was filtered and acidified using $5 \mathrm{~mL}$ of $10 \% \mathrm{HNO}_{3}$ solution. Three specimens were tested for each $\mathrm{Rm}$ CSC.

\section{Ex-vivo human vital tooth-culture model}

\section{Teeth sampling and culturing}

The samples were gathered upon a protocol approved by the institution's Commission for Medical Ethics (file number S54254). Fully impacted human third molars with their roots only partially formed, were collected immediately after extraction from five healthy young patients (14-18 years). A total of 10 teeth were used in this experiment. In the surgery room, the teeth were placed immediately after extraction in $50 \mathrm{ml}$ centrifuge tubes (VWR, Leuven, Belgium), containing Dulbecco's Modified Eagle Medium (DMEM; Gibco, Merelbeke, Belgium) supplemented with 10\% Fetal Bovine Serum (FBS; 
Gibco), 1\% penicillin-streptomycin (Gibco) and $1 \%$ amphotericin B (Gibco) (tooth-culture medium). The samples were brought to the cell-culture room within $4 \mathrm{~h}$ to proceed with the cell-culture experiments. Once in the cell-culture room, the periodontal ligament of the teeth was removed with a sterile \#15 scalpel blade (Swann Morton, Sheffield, UK). Then, all teeth were first rinsed with $70 \%$ ethanol (Hydral 70, VWR) for 1 min, followed by rinsing with sterile Phosphate Buffered Saline (PBS; Sigma Aldrich, St. Louis, MO, USA) for $1 \mathrm{~min}$, upon which they were placed in tooth-culture medium until further processed. After cleaning, the teeth were handled with sterile gauzes (Yibon Medical, Kuurne, Belgium) and soaked in tooth-culture medium to avoid desiccation. Six teeth ( $n=2$ per experimental group) were capped with the three experimental Rm-CSCs Exp_HEAA, Exp_GDM and Exp_HEAA/GDM, and kept in tooth-culture medium for $2 \mathrm{wk}$, as described below. Two teeth were kept as negative control (exposure without capping) and another two teeth were used as comparative commercial reference (TheraCal LC, Bisco).

\section{Ex-vivo human vital tooth-culture pulp-capping assay}

Once the teeth had been cleaned, a pulp-capping procedure was performed in sterile conditions and with the aid of $2.8 \mathrm{x}$ magnification loupes, similarly as done clinically. For the pulp-capping assay, we followed the protocol described by Téclès et al. (2005). Briefly, a class-V cavity (approximately $4 \times 4 \times 4$ mm) was cut using a sterile bur (1.1-mm diameter; Endo Access Bur Size 1, A 0164300001 00, Dentsply Sirona, Ballaigues, Switzerland) at high speed under copious irrigation with sterile saline (Fresenius Kabi, Bad Homburg, Germany). The pulp was exposed with a round carbide bur (1.0-mm diameter; H1SE.205.010, Komet, Lemgo, Germany) at low speed under abundant irrigation. Afterwards, the cavity was cleaned with sterile saline and gently dried with sterile cotton pellets.

The teeth were divided into five groups depending on the pulp-capping procedure carried out $(n=2)$ (Table 1): (1) Exp_HEAA, (2) Exp_GDM, (3) Exp_HEAA/GDM, (4) TheraCal LC (Bisco), and (5) exposure without capping as negative control.

All $\mathrm{Rm}$-CSCs were applied in a layer with a thickness of $1-2 \mathrm{~mm}$ following the manufacturer's recommendations. The cavity was restored with glass-ionomer cement (Fuji II LC Capsules, GC). Next, flowable composite (G-aenial Flo, GC) was applied on the occlusal surface, in which a sterilized stainless steel orthodontic wire (M form; ORMCO, Orange, CA, USA) was seated, followed by 40-sec light-curing using a light-curing unit with a light output of $1200 \mathrm{~mW} / \mathrm{cm}^{2}$ (Bluephase 20i, Ivoclar Vivadent, Schaan, Liechtenstein). The teeth were immediately hanged using the wire in separate wells of 24-well culture plates (Costar, Cambridge, MA, USA), each containing $1.5 \mathrm{ml}$ of tooth-culture medium to ensure generous exposure of the pulp tissue to the medium. The medium was refreshed every day and after two weeks the wire was removed, upon which the teeth were immediately placed in $4 \%$ paraformaldehyde (PFA) for two weeks to properly fix the tissue. 


\section{Micro-computed tomography $(\mu \mathrm{CT})$}

All teeth were scanned by means of a $\mu \mathrm{CT}$ scanner (Skyscan 1172, Bruker Skyscan, Kontich, Belgium) using the following parameters: $100 \mathrm{kV} / 100 \mu \mathrm{A} X$-ray source, $0.5-\mathrm{mm}$ aluminum filter, pixel size of 9.92 $\mu \mathrm{m}$, averaging frame of 7 , rotation step of $0.3^{\circ}$, random movement of 50 and $360^{\circ}$ rotation around the vertical axis. The raw data of all specimens was reconstructed using NRecon v.1.6.9.18 software (Bruker Skyscan) and the image processing and analysis were performed with FiJi ImageJ software ( $\mathrm{V}$ 1.52p) and CT-Analyser (CTAn v. 1.18.8.0, Bruker Skyscan). The 3D model rendering was made using CT Vox (CTvox, v.3.3.0, Bruker Skyscan).

\section{Histology and immunostaining}

The chemically fixed teeth were demineralized (4-6 weeks) with $10 \%$ formic acid (Chem-Lab Analytical, Zedelgem, Belgium) with the decalcifying solution refreshed every three days. The decalcification endpoint was determined by dental radiography (MINRAY, Soredex, Tuusula, Finland) and visual/tactile evaluation. After decalcification, the glass-ionomer and composite restorations were manually removed, upon which the teeth were subsequently immersed in water for $24 \mathrm{~h}$ and then dehydrated in ascending concentrations of ethanol (50\%, 70\%, 80\%, 95\% and $100 \%$ for $12 \mathrm{~h}$ each), followed by xylene (VWR) clearance. The teeth were next immersed in liquid paraffin $\left(56^{\circ} \mathrm{C}\right.$ melting point; Paraclean, Klinipath, Duiven, The Netherlands) for 24 h, prior to being embedded in paraffin blocks. Serial paraffin sections were cut with a thickness of 5-7 $\mu \mathrm{m}$ using a microtome (Microm HM 360 Microtome, Hyland Scientific, Stanwood, WA, USA), this from the level where the interaction of the pulp-capping agent with pulp tissue first appeared until any interaction was no longer visible.

Every six sequential sections, three sections were randomly selected for staining. One section was stained with Gill's III hematoxylin (Leica Microsystems, Diegem, Belgium) and with 1\% aqueous eosin solution (Leica Microsystems). The other two sections were selected for immunohistochemical characterization of collagen I (anti-collagen I antibody COL-1, ab6308, Abcam, Cambridge, UK) and nestin (anti-nestin antibody 10C2, ab22035, Abcam), respectively. The stained sections were examined using light microscopy (Axio Imager M2, Carl Zeiss Microscopy, Jena, Germany). The formation/presence of a fibrous band and mineralized foci around the exposed area was evaluated by two independent researchers.

\section{Statistical analysis}

A Kruskal-Wallis test (Bonferroni correction for multiple testing) at a significance level of $\alpha=0.05$ was selected for statistical analysis conducted using IBM SPSS Statistics (version 26; IBM, Chicago, IL, USA). 


\section{Results}

\section{Three-point bending flexural strength (Fig. 2)}

All experimental Rm-CSCs revealed a significantly higher $(p<0.05)$ flexural strength, without any mutual statistical significance, than TheraCal LC (Bisco).

\section{Shear bond strength to dentin (Fig. 3)}

All experimental Rm-CSCs revealed a significantly higher $(\mathrm{p}<0.05)$ shear bond strength to dentin, without any mutual statistical significance, than TheraCal LC (Bisco).

pH (Fig. 4a) and Ca release (Fig. 4b)

At $24 \mathrm{~h}$, Exp_HEAA revealed a significantly higher $(\mathrm{p}<0.05)$ mean $\mathrm{pH}$ value than the other experimental and reference Rm-CSCs without any mutual statistical significance. At 1 week, no significant difference in $\mathrm{pH}$ was measured for Exp_HEAA and Exp_HEAA/GDM (p>0.05). Both Rm-CSCs' pH remained significantly higher $(p<0.05)$ than that of Exp_GDM and TheraCal LC $(B i s c o)$ at 1 week.

At $24 \mathrm{~h}$, Ca release in particles per million $(\mathrm{ppm})$ was significantly higher $(p<0.05)$ for Exp_HEAA than for the other experimental and reference Rm-CSCs without any mutual statistical significance. At 1 week, Exp_HEAA and Exp_HEAA/GDM revealed significantly higher $(p<0.05)$ Ca release than Exp_GDM and TheraCal LC (Bisco). Comparing the 24-h versus 1-week Ca release, Ca release decreased for Exp_HEAA, while it increased for Exp_HEAA/GDM, with Ca release of Exp_GDM and TheraCal LC (Bisco) having remained almost equally low.

\section{Ex-vivo human vital tooth-culture pulp-capping assay}

\section{Micro-computed tomography ( $\mu \mathrm{CT}$ ) (Fig. 5)}

The teeth capped with the experimental Rm-CSC Exp_HEAA revealed the presence of radiopaque structures in the pulp area immediately underneath the pulp exposure after 2-week ex-vivo human vital tooth culturing. In contrast to the Exp_HEAA-capped teeth, $\mu \mathrm{CT}$ of the TheraCal LC-capped teeth did not reveal radiodensity increase of pulp tissue underneath the exposure that would indicate mineral foci formation.

\section{Histology and immunostaining (Figs. 6-8)}

Histology confirmed that for the teeth capped with the experimental Rm-CSC Exp_HEAA, the radiopaque zone, imaged by $\mu \mathrm{CT}$ (Fig. 5), stained more eosinophilically than the deeper pulp tissue (Figs. 6a,b and 7a,b). At higher magnification, the area immediately adjacent the pulp exposure appeared richer in cells, collagen fibrils and capillaries (Figs. 6b and 7b). 
The two teeth capped with the experimental Rm-CSC Exp_HEAA revealed the presence of mineral foci immediately adjacent the exposure area after 2-week ex-vivo human vital tooth culturing (Figs. 6a,b, 7a,b and 8a-d). The teeth capped with Exp_GDM and Exp_HEAA/GDM did not form mineralized foci two weeks after pulp capping (Table 2; Fig. 7c-f). Exp_HEAA stimulated the formation of mineral structures around the exposure site, which may eventually lead to the future formation of a mineralized bridge covering/healing the exposed region (Figs. 7a,b and 8a-d). At higher magnification, the area immediately adjacent the pulp exposure appeared rich in fibrinous tissue and mineral foci (Fig. 7b). However, when the teeth were capped with the reference Rm-CSC TheraCal LC (Bisco) or remained uncapped (control), no indications of mineral tissue formation, nor fibrinous tissue reaction were observed adjacent the exposed area [Figs. 7 (c-f) and 8e,f].

\section{Discussion}

This multi-parameter assessment showed that all three newly developed resin-modified calciumsilicate cements (Rm-CSCs) have a higher flexural strength and shear bond strength to dentin than the commercial Rm-CSC reference TheraCal LC (Bisco). Therefore, the first and second null-hypotheses that the new experimentally developed pulp-capping agents do not have improved flexural strength, nor increased shear bond strength to dentin, as compared to the commercial Rm-CSC TheraCal LC (Bisco), failed to be accepted. Furthermore, one Rm-CSC at $24 \mathrm{~h}$ and 1 week (Exp_HEAA) and another Rm-CSC at 1 week (Exp_HEAA/GDM) revealed a higher $\mathrm{pH}$ and Ca release than TheraCal LC (Bisco), by which also the third and fourth null-hypotheses failed to be accepted. Finally, also the fifth nullhypothesis that no pulp-healing initiation was expected upon pulp capping with the Rm-CSCs using the ex-vivo human vital tooth-culture model, failed to be accepted; the Exp_HEAA-capped teeth revealed the presence of mineral foci underneath the pulp exposure area already after 2-week ex-vivo human vital tooth culturing.

Bis-GMA (bisphenol A-glycidyl methacrylate) along with HEMA (2-hydroxyethyl methacrylate) is one of the most common combinations of monomers used in resin-based adhesive materials due to Bis-GMA's good physio-mechanical properties and HEMA's high wettability [19, 20, 22]. However, BisGMA is highly viscous, which limits double-bond conversion and requires the incorporation of diluent co-monomers to enhance polymerization conversion but also to increase filler-particle loading [21]. Moreover, the alleged potential presence of Bisphenol A (BPA) as an impurity or degradation product remains to raise concerns about the biosafety of Bis-GMA-containing formulations [23, 24]. Some studies found increased presence of BPA in patient's urine and saliva after dental procedures $[25,26]$. Regarding HEMA, this small hydrophilic monomer promotes water uptake, as well as it was shown to be cytotoxic towards human cells $[27,28]$. On the other hand, new alternative monomers from natural 
origin have recently been developed with potential to be used in dental materials $[21,29]$. The above sketched rationale is the main reason why we decided in this research to substitute Bis-GMA and HEMA, respectively, with UDMA (25wt\%), already used in resin-based dental materials, and FAM-401 (5wt\%). By doing so, we managed to maintain good wettability and hydrophilicity without losing physio-mechanical properties, as was shown by the 3-point bending flexural strength test. Furthermore, to achieve bonding potential to dentin the functional monomer 4-MET (10wt\%), providing ionic binding interaction with hydroxyapatite [30] was added to the Rm-CSC formulations, hereby having resulted in a significantly higher shear bond strength as compared to the reference RmCSC.

Successful vital pulp therapy depends, from a biological point of view, on 3 cornerstones: (1) a healthy patient with a good healing capacity; (2) an isolated and bacteria-free environment; and (3) a bioactive and biocompatible material which promotes human tooth-pulp cells to create a reparative dentin barrier to protect the pulp tissue from external noxious agents [11, 31, 32]. Furthermore, from a clinical point of view, the ideal pulp-capping agent should be user-friendly, enabling fast application and setting, the latter by preference on command, while it also should have good bonding capacity to dentin. This will enable the dentist, immediately after achieving pulp hemostasis, to place the pulpcapping material in preferentially 1 increment followed by the adhesive placement of the definitive direct restorative material that can co-polymerize with the Rm-CSC. In this sense, the development of a self-adhering resin modified calcium-silicate materials with adequate calcium release and mechanical strength would thus enable clinicians to perform vital pulp therapies in an efficient and fast manner.

TheraCal LC (Bisco) has relatively recently been marketed with the primary goal to offer dentists an easy to use pulp-capping agent that at least overcomes the handling shortcomings of resin-free hydraulic calcium-silicate cements (hCSCs). However, has shown that this Rm-CSC has still limitations, such as (1) relatively high cytotoxicity in contact with human tooth-pulp cells, (2) lack of bonding capacity to dentin, and (3) relatively poor Ca release $[13,17,33-37]$. Therefore, by conducting this exploratory research we presented three innovative Rm-CSC formulations with improved material properties of relevance concerning pulp-capping. Most promising appeared the Exp_HEAA Rm-CSC formulation. By adding the novel HEAA acrylamide monomer, higher hydrophilicity was achieved, combining a higher alkaline $\mathrm{pH}$ with facilitated $\mathrm{Ca}$ release from the TCS powder. This Rm-CSC formulation was proven to improve bioactivity, as was histologically evidenced by pulp-healing initiation through mineral foci formation.

Successful pulp capping is achievable when the pulp-capping agent possesses excellent biocompatibility towards human tooth-pulp cells [38-41]. In this sense, no direct biocompatibility testing was reported in this work. However, the cytotoxicity of the different experimental Rm-CSCs was tested towards human pulp fibroblasts in prior pilot experiments, having yielded excellent results. 
Nevertheless, a battery of biocompatibility tests, including animal experimentation, is mandatory before the materials can be tested in patients on their clinical effectiveness. These findings were confirmed by the ex-vivo human vital tooth-culture model, which was validated before [42-45]. No adverse pulp-tissue reaction was observed for any of the Rm-CSCs investigated; on the contrary, the Exp_HEAA Rm-CSC revealed histological indications of early reparative tissue formation with mineral foci.

Some of the limitations of the current research are that no evaluation of the biocompatibility and genotoxicity of the materials tested were done. Moreover, ion releasing materials are prone to suffer dissolution of the material which may imply weakening of the material and lack of radiopacity over time. In this way, future research will be conducted on the biocompatibility and the long-term mechanical properties of this kind of materials.

\section{Conclusion}

This correlative in-vitro and ex-vivo study revealed promising results for the developed experimental $\mathrm{Rm}$-CSCs in terms of mechanical strength and shear bond strength to dentin, as well as they provide an alkaline $\mathrm{pH}$ with facilitated Ca release. However, only Exp_HEAA revealed pulp-healing initiation in direct contact with human dental pulp tissue in the ex-vivo human vital tooth-culture model. This promising outcome for Exp_HEAA should be attributed to the combined use of (1) a novel hydrophilic acrylamide monomer, enabling sufficient polymerization while maintaining adequate hydrophilicity, with (2) the functional monomer 4-MET, possessing chemical bonding potential to dentin, and (3) tricalcium silicate powder to achieve an alkaline $\mathrm{pH}$ and to release $\mathrm{Ca}$ in a sufficient and controlled way. Notably improved performance was achieved as compared to the commercial reference Rm-CSC TheraCal LC (Bisco). Once the composition of the experimental Exp_HEAA Rm-CSC formulation has fully been optimized, further testing will include biocompatibility and genotoxicity testing, along with animal experimentation according to the respective ISO guidelines, this towards the commercial introduction of a novel resin-modified pulp-capping agent.

\section{Acknowledgments}

This work was supported by the FWO research grant G.0893.15 and by the Japan Society for the Promotion of Science (JSPS) grant numbers JP18K17068 and JP18K09597.

We would like to thank all staff members of the Oral and Maxillofacial Surgery (MKA) of UZ Leuven (University Hospitals Leuven) for their help to collect freshly extracted human third molars. We also want to acknowledge the dental manufacturers Bisco for providing the commercial materials. 


\section{Conflict of Interest statement}

The authors have explicitly declared to have received public grants (FWO and JSPS) in relationship with this work. 


\section{References}

[1] F. Schwendicke, F. Brouwer, A. Schwendicke, S. Paris, Clin Oral Investig, 20 (2016) 11211132.

[2] R. Emara, K. Elhennawy, F. Schwendicke, J Dent, 77 (2018) 18-36.

[3] P. Sangwan, A. Sangwan, J. Duhan, A. Rohilla, Int Endod J, 46 (2013) 3-19.

[4] M. Torabinejad, M. Parirokh, P.M.H. Dummer, Int Endod J, 51 (2018) 284-317.

[5] B.I. Suh, R. Yin, M. Cannon, D. Martin, International Patent A61k33/42;A61K33/42, vol. 1 (2008).

[6] M.G. Gandolfi, F. Siboni, C. Prati, Int Endod J, 45 (2012) 571-579.

[7] U. Schröder, J Dent Res, 64 Spec No (1985) 541-548.

[8] M.G. Gandolfi, G. Spagnuolo, F. Siboni, A. Procino, V. Rivieccio, G.A. Pelliccioni, C. Prati, S. Rengo, Clin Oral Investig, 19 (2015) 2075-2089.

[9] J.F. Siqueira, Jr., H.P. Lopes, Int Endod J, 32 (1999) 361-369.

[10] D. Ricucci, S. Loghin, L.M. Lin, L.S. Spångberg, F.R. Tay, J Dent, 42 (2014) 1156-1170.

[11] D. Ricucci, J.F. Siqueira, Jr., Y. Li, F.R. Tay, J Dent, 86 (2019) 41-52.

[12] H. Alzraikat, N.A. Taha, D. Qasrawi, M.F. Burrow, Dent Mater J, 35 (2016) 881-887.

[13] N.Z. Arandi, T. Rabi, Int J Dent, 2018 (2018) 3484653.

[14] M.G. Gandolfi, F. Siboni, T. Botero, M. Bossu, F. Riccitiello, C. Prati, J Appl Biomater Funct Mater, 13 (2015) 43-60.

[15] E.A. Bortoluzzi, L.N. Niu, C.D. Palani, A.R. El-Awady, B.D. Hammond, D.D. Pei, F.C. Tian, C.W. Cutler, D.H. Pashley, F.R. Tay, Dent Mater, 31 (2015) 1510-1522.

[16] T. Giraud, P. Rufas, F. Chmilewsky, C. Rombouts, J. Dejou, C. Jeanneau, I. About, J Endod, 43 (2017) 1104-1110.

[17] C. Jeanneau, P. Laurent, C. Rombouts, T. Giraud, I. About, J Endod, 43 (2017) 2074-2080.

[18] K. Yoshihara, N. Nagaoka, T. Okihara, M. Irie, A. Matsukawa, M.S. Pedano, Y. Maruo, Y. Yoshida, B. Van Meerbeek, J Mater Chem B, 8 (2020) 5320-5329.

[19] E. Asmussen, A. Peutzfeldt, Dent Mater, 14 (1998) 51-56.

[20] K.L. Van Landuyt, J. Snauwaert, J. De Munck, M. Peumans, Y. Yoshida, A. Poitevin, E. Coutinho, K. Suzuki, P. Lambrechts, B. Van Meerbeek, Biomaterials, 28 (2007) 3757-3785. [21] A.P. Fugolin, A.B. de Paula, A. Dobson, V. Huynh, R. Consani, J.L. Ferracane, C.S. Pfeifer, Dent Mater, 36 (2020) 884-892.

[22] L. Hitmi, D. Bouter, M. Degrange, Dent Mater, 18 (2002) 503-511.

[23] S. De Nys, E. Putzeys, P. Vervliet, A. Covaci, I. Boonen, M. Elskens, J. Vanoirbeek, L. Godderis, B. Van Meerbeek, K.L. Van Landuyt, R.C. Duca, Sci Rep, 8 (2018) 6981.

[24] P. Vervliet, J.V. Den Plas, S. De Nys, R.C. Duca, I. Boonen, M. Elskens, K.L. Van Landuyt, A. Covaci, Toxicology, 420 (2019) 1-10.

[25] K.L. Van Landuyt, T. Nawrot, B. Geebelen, J. De Munck, J. Snauwaert, K. Yoshihara, H. Scheers, L. Godderis, P. Hoet, B. Van Meerbeek, Dent Mater, 27 (2011) 723-747.

[26] E. Putzeys, S.M. Cokic, H. Chong, M. Smet, J. Vanoirbeek, L. Godderis, B. Van Meerbeek, K.L. Van Landuyt, R.C. Duca, J Sep Sci, 40 (2017) 1063-1075.

[27] R. Perduns, J. Volk, P. Schertl, G. Leyhausen, W. Geurtsen, Dent Mater, 35 (2019) 501510.

[28] P. Schertl, J. Volk, R. Perduns, K. Adam, G. Leyhausen, A. Bakopoulou, W. Geurtsen, Dent Mater, 35 (2019) 144-155.

[29] S.-F. Koelewijn, D. Ruijten, L. Trullemans, T. Renders, P. Van Puyvelde, H. Witters, B.F. Sels, Green Chem, 21 (2019) 6622-6633. 
[30] K. Nagakane, Y. Yoshida, I. Hirata, R. Fukuda, Y. Nakayama, K. Shirai, T. Ogawa, K. Suzuki, B. Van Meerbeek, M. Okazaki, Dent Mater J, 25 (2006) 645-649.

[31] L. Bjorndal, S. Simon, P.L. Tomson, H.F. Duncan, International Endodontic Journal, 52 (2019) 949-973.

[32] P.M.H. Dummer, V. Franco, G. Gambarini, D. Orstavik, L. Tjaderhane, J. Whitworth, H.F. Duncan, K.M. Galler, P.L. Tomson, S. Simon, I. El-Karim, R. Kundzina, G. Krastl, T.

Dammaschke, H. Fransson, M. Markvart, M. Zehnder, L. Bjorndal, Ese, Int Endod J, 52 (2019) 923-934.

[33] M.G. Gandolfi, F. Siboni, C. Prati, International Endodontic Journal, 45 (2012) 571-579. [34] E.A. Bortoluzzi, L.N. Niu, C.D. Palani, A.R. El-Awady, B.D. Hammond, D.D. Pei, F.C. Tian, C.W. Cutler, D.H. Pashley, F.R. Tay, Dental Materials, 31 (2015) 1510-1522.

[35] T. Giraud, P. Rufas, F. Chmilewsky, C. Rombouts, J. Dejou, C. Jeanneau, I. About, Journal of Endodontics, 43 (2017) 1104-1110.

[36] B.W. Nilsen, E. Jensen, U. Ortengren, V.B. Michelsen, European Journal of Oral Sciences, 125 (2017) 183-194.

[37] X. Li, M.S. Pedano, B. Camargo, E. Hauben, S. De Vleeschauwer, Z. Chen, J. De Munck, K. Vandamme, K. Van Landuyt, B. Van Meerbeek, Dent Mater, 34 (2018) 1410-1423.

[38] M.S. Pedano, X. Li, K. Yoshihara, K.V. Landuyt, B. Van Meerbeek, Materials (Basel), 13 (2020).

[39] M.S. Pedano, X. Li, S. Li, Z. Sun, S.M. Cokic, E. Putzeys, K. Yoshihara, Y. Yoshida, Z. Chen, K. Van Landuyt, B. Van Meerbeek, Dent Mater, 34 (2018) 797-808.

[40] J.L. Sanz, L. Forner, C. Llena, J. Guerrero-Gironés, M. Melo, S. Rengo, G. Spagnuolo, F.J. Rodríguez-Lozano, J Clin Med, 9 (2020).

[41] M. Collado-Gonzalez, D. Garcia-Bernal, R.E. Onate-Sanchez, P.S. Ortolani-Seltenerich, T. Alvarez-Muro, A. Lozano, L. Forner, C. Llena, J.M. Moraleda, F.J. Rodriguez-Lozano, Int Endod J, 50 Suppl 2 (2017) e19-e30.

[42] O. Tecles, P. Laurent, V. Aubut, I. About, Journal of Biomedical Materials Research B Applied Biomaterials, 85 (2008) 180-187.

[43] P. Laurent, J. Camps, I. About, Int Endod J, 45 (2012) 439-448.

[44] M.S. Pedano, X. Li, C. Jeanneau, M. Ghosh, K. Yoshihara, K. Van Landuyt, I. About, B. Van Meerbeek, J Dent, 86 (2019) 33-40.

[45] M.S. Pedano, X. Li, B. Camargo, E. Hauben, S. De Vleeschauwer, K. Yoshihara, K. Van Landuyt, Y. Yoshida, B. Van Meerbeek, Dent Mater, 36 (2020) 512-526. 
Table 1. List of the resin-modified calcium-silicate cements (Rm-CSCs) investigated, their composition and their application mode.

\begin{tabular}{|c|c|c|}
\hline Rm-CSC (company) & Composition (wt\%) & Application mode \\
\hline Exp_HEAA & $\begin{array}{l}\text { TCS powder (37\%) } \\
\text { UDMA (25\%) } \\
\text { HEAA }(20 \%) \\
\text { 4-MET }(10 \%) \\
\text { FAM- } 401(5 \%) \\
\mathrm{ZrO}_{2}(3 \%)\end{array}$ & \\
\hline Exp_GDM & $\begin{array}{l}\text { TCS powder (37\%) } \\
\text { UDMA (25\%) } \\
\text { GDM (20\%) } \\
\text { 4-MET (10\%) } \\
\text { FAM- } 401(5 \%) \\
\mathrm{ZrO}_{2}(3 \%)\end{array}$ & $\begin{array}{l}\text { Apply Rm-CSC in a layer of 1-2 mm; } \\
\text { photo-polymerize for } 20 \mathrm{~s}\end{array}$ \\
\hline Exp_HEAA/GDM & $\begin{array}{l}\text { TCS powder (37\%) } \\
\text { UDMA (25\%) } \\
\text { HEAA (10\%) plus GDM (10\%) } \\
\text { 4-MET (10\%) } \\
\text { FAM- } 401(5 \%) \\
\mathrm{ZrO}_{2}(3 \%)\end{array}$ & \\
\hline TheraCal LC (Bisco, Schaumburg, IL, USA) & $\begin{array}{l}\text { *Type III Portland cement (30-50\%) } \\
\text { Polyethylene glycol dimethacrylate (10-30\%) } \\
\text { Barium zirconate (1-10\%) }\end{array}$ & \\
\hline
\end{tabular}

HEAA = N-(2-hydroxyethyl) acrylamide; FAM-401 = N,N'-\{[(2-acrylamido-2-[(3-acrylamidopropoxy)methyl]propane-1,3diyl)bis(oxy)]bis-(propane-1,3-diyl)\}c diacrylamide; GDM = glycerol dimethacrylate (GDM); TCS = tricalcium silicate; UDMA = urethane dimethacrylate; $\mathrm{ZrO}_{2}=$ zirconium dioxide; 4-MET = 4-methacryloxyethyl trimellitic acid; *According to technical information provided by the manufacturer. 
Table 2. Formation of mineral foci adjacent the pulp-exposure area.

\begin{tabular}{lcc}
\hline \multirow{2}{*}{ Rm-CSC } & \multicolumn{2}{c}{ 2-Week ex-vivo human vital tooth culturing } \\
& Teeth total $(\mathrm{n})$ & \begin{tabular}{c} 
Teeth presenting mineral foci (\%) \\
\hline Exp_HEAA
\end{tabular} \\
Exp_GDM & 2 & $2(100 \%)$ \\
Exp_HEAA/GDM & 2 & $0(0 \%)$ \\
TheraCal LC & 2 & $0(0 \%)$ \\
Control (exposure without pulp-capping agent) & 2 & $0(0 \%)$ \\
& & $0(0 \%)$ \\
\hline
\end{tabular}




\section{FIGURE LEGENDS}

Figure 1. 2D and 3D molecular structures of the experimental hydrophilic monomers HEAA and GDM investigated.

Figure 2. Flexural strength of Rm-CSCs, as determined by 3-point bending. Different letters indicate statistically significant difference between the Rm-CSCs tested ( $p<0.05)$.

Figure 3. Shear bond strength of Rm-CSCs to dentin. Different letters indicate statistically significant difference between the Rm-CSCs tested $(p<0.05)$.

Figure 4. $\mathrm{pH}$ in (a) and calcium ( $\mathrm{Ca}$ ) release in (b) of $\mathrm{Rm}-\mathrm{CSCs}$, as determined at $24 \mathrm{~h}$ and 1 week. Different letters indicate statistically significant difference between the Rm-CSCs tested $(p<0.05)$.

Figure 5. Micro-CT ( $\mu \mathrm{CT}$ ) imaging of two teeth capped for 2 wk by Exp_HEAA in (a-c) and one tooth capped for $2 \mathrm{wk}$ by TheraCal LC (Bisco) in (d) using the human vital tooth-culture model. The $\mu \mathrm{CT}$ scan (a) and 3D reconstruction (b) of the Exp_HEAA-capped tooth revealed a radiodensity increase in comparison with the area of the pulp tissue underneath the exposure, most likely representing mineral foci formation (arrow and interrupted line). (c) Micro-CT of another Exp_HEAA-capped tooth presenting likewise with a radiodensity increase of pulp tissue underneath the exposure (arrow and stripped line). (d) Micro-CT scan of the TheraCal LC-capped tooth showing no radiodensity increase of pulp tissue underneath the exposure that would indicate mineral foci formation. $(E=$ enamel; $D=$ dentin; $M=$ pulp-capping material).

Figure 6. Pulp-tissue reaction of three representative teeth after 2 wk exposure in the human vital tooth-culture model, with histological micrographs of pulps that were capped by Exp_HEAA in $(\mathbf{a}, \mathbf{b})$, Exp_GDM in (c,d), and Exp_HEAA/GDM in (e,f). (a) Overview micrograph showing the pulp exposure and capping with Exp_HEAA. An amorphous, eosinophilic layer was detected underneath the capped area. Beneath this layer, many mineral foci (hand pointers) were observed in the pulp tissue immediately adjacent to the exposure site. (b) Higher magnification of the rectangle in (a), identifying accumulation of 20-50 $\mu \mathrm{m}$ mineral foci within the pulp tissue next to the capped site (hand pointers). (c) Overview micrograph of a tooth capped with Exp_GDM. (d) Higher magnification of the rectangle in (c), showing absence of an eosinophilic layer, nor mineral foci formation in the capped area. (e) Overview micrograph of a tooth capped with Exp_HEAA/GDM, revealing remnants of the Rm-CSC in the capped area. (f) Higher magnification of the rectangle in (e); no mineral foci, nor fibrinous tissue reaction was observed. $(D=$ dentin; $P=$ pulp tissue $)$. 
Figure 7. Pulp-tissue reaction of another tooth capped for 2 wk by Exp_HEAA in $(a, b)$, TheraCal LC (Bisco) in (c,d) and Control (exposure without capping) (e, f) using the human vital tooth-culture model. (a) Overview micrograph showing remnants of Exp_HEAA above the exposure site. An amorphous, eosinophilic layer was detected underneath the capped area. Beneath the layer of debris, mineral foci were observed in the pulp tissue adjacent to the exposure site (hand pointers). (b) Higher magnification of the rectangle in (a), showing the presence mineral foci within the pulp tissue next to the capped site (hand pointers). (c) Overview micrograph of a tooth capped by TheraCal LC (Bisco). (d) Higher magnification of the rectangle in (c) did not reveal any reaction in the pulp tissue around the exposure area; no mineral foci, nor fibrinous tissue reaction was observed. (e) Micrograph of a control tooth (exposure without capping). (f) Higher magnification of the rectangle in (e), showing absence of tissue reaction to the pulp exposure. No mineral foci or cellular migration can be seen in the exposed area. $(\mathrm{D}=$ dentin; $\mathrm{P}=$ pulp tissue).

Figure 8. Immunohistochemistry of two teeth capped for 2 wk by Exp_HEAA in (a-d) and TheraCal LC (Bisco) in (e,f) using the human vital tooth-culture model. (a) Overview micrograph of an Exp_HEAAcapped tooth stained with nestin, showing remnants of Exp_HEAA above the exposure site. Immediately underneath the exposure site, some mineral foci were observed in the pulp tissue adjacent to the exposure site. (b) Higher magnification of the rectangle in (a), showing the presence of 20-50 $\mu \mathrm{m}$ mineral foci that were strongly stained with nestin (hand pointers). (c) Overview of another Exp_HEAA-capped tooth stained with collagen I. (d) Higher magnification of the rectangle in (c), showing the presence of mineral foci (hand pointers). In this case, the foci were stained less intense than with nestin, indicating the specificity of the mineral structures formed as dentin, in opposition to bone, is more intensively stained with nestin than with collagen I. (e) Histological slide of a TheraCal LC-capped tooth stained with nestin. Remnants of the Rm-CSC can be seen above the exposure side, while no fibrinous layer, nor mineral foci were observed. (f) Higher magnification of the rectangle in (e) did not reveal any reaction in the pulp tissue next to the exposure area (interrupted line and hand pointers); no mineral foci, nor fibrinous tissue reaction was observed. ( $P=$ pulp tissue). 